\title{
LASER SURGERY FOR GLÁUCOMA: MINIMALLY INVASIVE THERAPY
}

\author{
JAMES A. McALLISTER \\ Windsor
}

The object of treating patients with glaucoma is to preserve vision. The pathological mechanisms resulting in glaucoma are varied and alter the approach to management. In general, treatment is aimed at lowering intraocular pressure to a level believed to be safe for that particular eye at that stage of disease.

Therapy is aimed at reducing aqueous production or at improving outflow either through the existing outflow pathway or by alternatively created drainage channels. In no other area of ophthalmology have there been more varied attempts at finding a cure.

In discussing the use of lasers in treating glaucoma it would be wrong to assume that they cannot be invasive to varying degrees, but they have achieved some reduction in the risk of ocular damage in certain situations. For example, laser iridectomy with the neodymium:YAG laser has achieved the creation of a functional opening in iris tissue in angle closure disease without incising the surface tissue of the eye. In most cases this facilitates the management of this disease and certainly aids prevention when used prophylactically in the fellow eye. Lasers perhaps represent a concept of a more precise and less traumatic method of performing certain surgical tasks without necessarily invading the integrity of the globe. This is certainly their image in the mind of the public, though to some extent it also exists in our minds as surgeons in contemplating their use.

We could, however, hardly consider neodymium:YAG cyclophotocoagulation and iridectomy in the same breath, even though both use infrared light at $1064 \mathrm{~nm}$ and only the press of a switch is required to convert Q-switched pulses to longer free-running thermal emission. Cyclo YAG follows in the footsteps of cyclocryotherapy as another weapon against refractory disease, and although the globe remains visibly unperforated the energy levels delivered to the eye, deep to the surface, are sufficient to produce extensive tissue damage to the target zone as well as a variable degree of undesirable inflammatory response. Its place in therapy can be defended by virtue of

Correspondence to: Mr. J. A. McAllister, FRCS, FCOphth, Princess Margaret Hospital, Osborne Road, Windsor, Berks SL4 3SJ, UK. the limited options currently available for the cases for which it is offered. These options usually mean either accepting that no further treatment is possible or using drainage implant tubes. The latter can be effective but are undeniably invasive and bring with them their own range of complications. Cyclo YAG does not compete for acceptability with tube implants, but there are situations where each can contribute to management and be equally helpful, as well as producing significant damage to intraocular integrity.

Leaving aside the method of reduction of aqueous production by laser (remembering that the least invasive therapy for producing this effect is, of course, the medical one of aqueous suppressants), most of the other efforts in developing and designing lasers for glaucoma in the eye have been concentrated on either improving existing outflow pathways or, more commonly, attempting to create alternative drainage channels from the eye.

Despite the 16 years that have passed since its original description, the exact contribution of argon laser trabeculoplasty to visual survival in glaucoma patients is still unclear. It is unarguable that it was a brave attempt to alter the resistance to outflow of eyes with open angle glaucoma and reduced filtration. It has also led indirectly to a greater understanding of the pathophysiology that occurs in the structures of the angle of the eye, because of attempts to understand how a trabeculoplasty itself causes its effects and why it sometimes works and sometimes fails. Laser trabeculoplasty, because of the necessity of seeing angle structures, has also encouraged the clinical assessment of angles by routine gonioscopy aided by the small four-mirror instruments not requiring contact solutions. This in turn has helped us understand the variations in anterior chamber angle that occur amongst the population. At the same time as laser trabeculoplasty was being studied, research on cell populations in the trabecular meshwork and the behaviour of the angle structures in the ageing eye has furthered our knowledge of the disease process of glaucoma itself. I think it would be fair to say that argon laser trabeculoplasty is minimally invasive and does, in perhaps $60-70 \%$ of cases, result in a lowering of intraocular pressure. 
We know that argon laser trabeculoplasty does not replace filtering procedures and that its effect is more akin to adding an additional medication to the eye, with pressure lowering of 6 or $7 \mathrm{mmHg}$ being the usual maximum. In those cases where this degree of pressure lowering is sufficient when added to other therapy, visual loss may well be halted for a variable period of time. I think, though, that we are all concerned that the use of the procedure might in some cases delay conventional surgery once visual loss is occurring. However, we have perhaps been too ready to denounce argon laser trabeculoplasty because of its modest contribution and in the light of its possible deleterious effect on subsequent filtering surgery; there are patients who do remarkably well for long periods after treatment and it should not lightly be dismissed.

I believe De Weckar first described 'la cicatrice à filtration' in 1882 in an attempt to control glaucoma, and the number of different procedures created since that time is considerable. From full-thickness procedures such as the Scheie and trephine to the partial-thickness sclerostomies generally (though not globally) used today, the same external route from the eye was the intention. It should be remembered, though, that John Cairns' concept of trabeculectomy did not imply this pathway as its attempted goal.

The current partial-thickness or guarded sclerostomy could be said to be less invasive than full-thickness procedures in that it provides greater protection to the deeper layers of the eye. Infection and conjunctival cysts are late complications of the full-thickness procedure. Efforts at producing a patent fistula that remains functional over many years are often frustrated by the cellular reaction of the eye to the injury of surgical trauma and subsequent scar formation. This healing process is compounded if tissue has been altered in its response prior to surgery by medication or by additional medication subsequently. We have become aware of the other factors that affect the success of surgery, in particular race, age and the alteration of tissue response by previous surgical procedures, and these all affect the short-term and long-term success of filtration. Knowledge of the processes involved in the inflammatory and healing responses of the eye have led to the development of anti-metabolic drugs that may enhance the effectiveness of these procedures, not only in the atrisk eye but also by improving the survival and pressurelowering effect after surgery in previously virgin eyes. Undesirable healing may be the biggest challenge to modern filtering surgery, but of course there are a number of real complications of opening the eye which still lead us to search for less invasive approaches whilst attempting to maintain or even improve on effective pressure lowering.

To date no laser method has reached a level of development and reliability which challenges the success rate of the carefully performed partial-thickness sclerostomy known as trabeculectomy. Indeed, although with this operation there are incisions into the eye with the potential for subsequent risks, in general the reliability and absence of complications in such surgery has led to its evaluation as potentially the primary treatment for managing open angle glaucoma. Perhaps invasiveness in the eye is therefore in a different context to invasiveness in general surgery, where laparoscopic cholecystectomy is seen as a great step forward in reducing post-operative morbidity or where closed surgery on knee joints using fibre-optic technology has revolutionised the results of meniscectomy. Perhaps eye surgeons have by definition always been less invasive because of the small size of the globe and the necessity for small incisions; ambulatory trabeculectomy is by no means unreasonable provided all the necessary follow-up arrangements are established.

Opening up the outflow channels by reflected laser light through gonio lenses has been investigated over a period of nearly two decades by Krasnov, Bechman, Fankhauser and others, and although visible and histologically detectable openings can be created which penetrate into Schlemm's canal it is rare for these openings to be maintained and functional. Recent studies by Melamed and Epstein working from Boston have demonstrated fibronectin deposition in the laser crater, both along its walls and at the fibrin clot, within 1 hour of treatment and this, along with collapse of the canal itself, is postulated as a reason for the failure of this technique. Full-thickness laser sclerostomies perhaps present a greater challenge and have been attempted with a variety of lasers with variable degrees of success. March used a neodymium:YAG laser, aiming Q-switched pulses through a specially designed contact lens to punch openings $20 \mu \mathrm{m}$ in diameter into the subconjunctival space. This technique seemed to require significant energy levels and his results have been difficult to reproduce. More recently dye lasers have been investigated, primarily by Melamed and colleagues in Tel Aviv. Their technique is also internal via a gonio lens using a $590 \mathrm{~nm}$ red pulsed dye laser aiming at the corneoscleral junction, but laser absorption is enhanced to produce openings with smaller energy levels by iontophoresing reactive black dye through the conjunctival surface. Removal of the tissue product in a closed space seems to be a problem with this approach, but filtering blebs have been created. Clinical studies have yet to identify the effectiveness of this externally non-invasive approach or to identify its potential shortcomings.

Holmium:YAG laser sclerostomy and its development is an example of reasoned thinking in an attempt to create a canal with minimal disturbance to the eye from an external approach. Using a wavelength near the infrared $(2.1 \mu \mathrm{m})$ and a long $300 \mu \mathrm{s}$ pulse, this laser can theoretically cauterise the edges of the created channel, the scleral tissue of which is vaporised as the channel is formed. A subconjunctival track is required with a small incision away from the site of treatment and the gold-tipped probe, with a $90^{\circ}$ reflective mirror at its tip, is gently introduced in the subconjunctival space to a point adjacent to the limbus. The exact position and angulation are quite critical, but in almost all cases filtration is readily achieved, allowing an ambulant day case approach. We must still call this technique invasive because of the disturbance of the 
anterior segment, and indeed this allows for the potential hazards associated with a full-thickness procedure such as a Scheie. Histological examination shows that the walls of the channel are more precise than with a surgical procedure, but problems with iris obstruction and Descemet's flaps internally still present difficulties and the smaller $300 \mu \mathrm{m}$ openings are prone to subsequent failure by subconjunctival healing. Studies with anti-metabolites may improve the success rate, which is currently in the $70 \%$ range.

Another external laser approach has been demonstrated as possible with the excimer laser. Its ultraviolet wavelength at $190 \mathrm{~nm}$ can remove layers of tissue with little or no associated damage. This allows for removal of most of the juxtacanalicular tissue (the main area of outflow resistance) until aqueous percolates through the base of the cavity without the anterior chamber itself being visibly invaded. With this technique a proper conjunctival flap is required before positioning the laser.

Finally, Fankhauser in Berne has been evaluating an internal approach using laser energy delivery through a $200 \mu \mathrm{m}$ silicon fibre encased in a 20 gauge needle (to provide rigid support) which passes through a paracentesis tract across the anterior chamber, previous filled with Healon into the opposite angle. Through this system, which he describes as an optico-mechanical approach, free-running and continuous-wave neodymium:YAG laser light is transmitted into the angle tissue. This results in tissue vaporisation and the formation of a channel without the excessive energy levels needed by the March technique.

The goal of all these laser approaches is the same, and they must be put to the same stringent tests as have been applied by virtue of the weight of clinical experience with trabeculectomy. Firstly, they must be shown in animal studies to be possible and apparently safe. Secondly, careful trials are required to extend awareness of safety and exact techniques. Thirdly, and this is the most difficult criterion to satisfy, they must withstand the test of time in comparison with existing surgical filtration methods. We must not presume that a new technique, whether it be with a laser or not, is necessarily an advantage, but it must be open to consideration as to whether producing filtration by an alternative method to trabeculectomy can make a genuine contribution to preventing patients suffering loss of vision as a result of glaucoma. The laser techniques described above, including laser trabeculoplasty, need not necessarily be dismissed as unsuccessful if they need additional medication, provided the treatment goal is achieved of successfully lowering the intraocular pressure to a level which maintains, or is believed to maintain, vision and stable visual fields. We are only at the beginning of laser surgery for glaucoma and the prospects remain exciting. 